\title{
Load Dependent Dynamic Path Selection in Multi-Radio Hybrid Wireless Mesh Networks
}

\author{
Raja Farrukh Ali and Adnan K. Kiani \\ School of Electrical Engineering and Computer Science, \\ National University of Sciences and Technology, \\ Islamabad, Pakistan. \\ Email: \{farrukh.ali, adnan.khalid\}@ seecs.edu.pk
}

\author{
Asad Amir Pirzada \\ College of Aeronautical Engineering, \\ National University of Sciences and Technology, \\ Islamabad, Pakistan. \\ Email: asad-cae@nust.edu.pk
}

\begin{abstract}
Hybrid Wireless Mesh Networks provide improved connectivity and reliability over traditional wireless networks. However due to rapidly changing traffic patterns, such networks are more likely to experience congestion which leads to data loss. Inability to differentiate between congested links and select best possible paths dynamically can be very expensive in a rapidly changing network topology. This paper proposes D-WCETT (Dynamic Weighted Cumulative Expected Transmission Time), an enhanced version of the WCETT routing metric, with focus on forming routes based upon network load information. The proposed routing metric dynamically selects paths with least level of congestion by taking into consideration locally available queue information. We present an evaluation of our implementation via extensive simulations and results indicate that the proposed metric significantly outperforms its predecessor by virtue of its ability to distinguish and dynamically select least congested paths in a multi-radio environment.
\end{abstract}

Keywords-Wireless mesh networks, multi-radio, routing metric, load aware routing, performance

\section{INTRODUCTION}

Wireless Mesh Networks provide robust and reliable wireless communication with minimal cost. Their dynamic, selforganizing and self-configuring nature enables nodes to establish and maintain mesh connectivity among themselves. A Wireless Mesh Network (WMN) consists of Mesh Routers and Mesh Clients in which Mesh Routers form the static network backhaul, possess better power resources and are equipped with multiple radios. They also provide gateway functionality by connecting the WMN to other networks. Mesh Clients are mobile nodes having limited battery resources and are mostly equipped with a single radio. They extend the reach of the network owing to their mobility but are dependent on Mesh Routers for their connectivity. WMNs are of three main types [1]; Infrastructure WMN consists of static Mesh Routers with Mesh Clients connected only via a single hop and devoid of any packet forwarding functionality. Client WMN represents a true Mobile Adhoc Network (MANET) as it consists of only Mesh Clients which communicate among each other directly and perform routing and configuration functions themselves. Hybrid WMN merges the connectivity model of Infrastructure and Client networks in which both Mesh Routers and Clients are involved in routing and packet forwarding and Mesh Clients can gain access to the network via multiple client hops.

A Hybrid WMN presents the most generic and prevalent type of mesh network. Multi-radio nodes can significantly enhance the capacity of the network by utilising the multiple orthogonal channels available to them and overcome limitation of half-duplex single radio nodes. Despite their widespread use, Hybrid WMNs face challenges in their deployment. Mobility poses a complex problem as it results in frequent route disruption and requires significant efforts for route maintenance under dynamic conditions. Moreover contention for wireless medium among nodes results in intrachannel and inter-channel interference. In order to overcome these limitations, routing protocols are constantly evolving to support mobility, reduce interference and establish reliable routes.

Routing protocols are designed keeping in view the networks in which they are to be employed. While a static network aims at improving the performance of individual transfers, a mobile network needs a protocol which can effectively maintain connectivity. A routing protocol employs a routing metric to select best possible paths between nodes. Routing metrics can be based on factors such as traffic, energy, topology and mobility [2] with hop count, delay, packet loss ratio, signal strength being a few examples. Routing protocols for wireless adhoc networks have used hop count as the default metric but it has been shown [3], [4] that shortest-path routing is not the most suitable metric as it tends to favor longer but slower paths. Subsequently proposed enhancements have used packet loss and channel interference among other factors that a routing protocol should consider in order to find better links. Such metrics tend to decrease path length, end-to-end delay and make effective utilization of available network bandwidth by reducing co-channel interference.

Hybrid WMNs however present a case where changing network topology adds another dimension to existing metric considerations. A routing metric should be able to adapt itself according to the dynamic nature of the network and its measurements should be reflective of the current network conditions. As will be shown later, a number of routing metrics designed for multi-hop multi-radio networks fail to meet this requirement. While employing multiple factors to make better overall judgement, they usually associate a tunable factor to alternate between different link quality indicators. This tunable factor cannot be changed dynamically and hence always gives a pre-determined weight to its constituent path functions. There is a need to make routing metrics adaptable to current network 
conditions which should enable them to increase the routing efficiency in a network.

In this paper we present Dynamic Weighted Cumulative Expected Transmission Time (D-WCETT) metric. The key contributions of this paper include a routing metric with the ability to select least congested links along a path by taking into consideration the interface queue length of the link. Moreover this selection is dynamic and is reflective of the prevalent load level of a link. Since this information is locally available at the node, it also does not incur any additional communication overhead. D-WCETT has been implemented in a multi-radio Hybrid WMN and has been evaluated at different mesh client speeds, packet transmission rates and traffic loads. Results indicate significantly better performance of the DWCETT metric with dynamic path selection over WCETT. This improved performance is attributed to the integration of the metric with the contemporary network load.

The rest of the paper is organized as follows. Section II discusses relevant related work in the field of routing metrics. The proposed routing metric D-WCETT is presented in Section III. Simulation results are analysed in Section IV. Section V concludes the paper.

\section{RELATED WORK}

Hop count is the most common metric used in adhoc routing protocols. However minimum hop count favors longer links and fails to consider important parameters such as transmission rates and link quality, consequently resulting in non-optimal performance [2]-[4].

Expected Transmission Count (ETX) [3] establishes a path depending upon the expected number of MAC layer transmissions which will result in delivering a packet successfully.

$$
E T X=\frac{1}{d_{f} \cdot d_{r}}
$$

ETX uses probe packets for its calculation such that probe packets successfully received from a neighboring node indicate the reverse delivery ratio $\left(d_{r}\right)$ whereas forward delivery ratio $\left(d_{f}\right)$ is measured through packets successfully received by a neighbor. These ratios are then used to calculate the probability of a successful transmission. Although ETX performs better than hop count, its limitations include inability to differentiate between links of different bandwidths and susceptibility to cochannel interference in multi-radio networks.

The Weighted Cumulative Expected Transmission Time (WCETT) [4] was developed specifically in the context of multi-radio networks. The main component of this metric is ETT which builds upon the ETX metric by incorporating packet size $(S)$ and bandwidth of the link $(B)$ and denotes the expected time required to successfully send a packet.

$$
E T T=E T X \cdot \frac{S}{B}
$$

The purpose of using multi-radio nodes is to maximise the available bandwidth by using multiple orthogonal channels available in the spectrum. It is desired that a packet uses multiple channels along a path such that all channels are equally utilized. A path which is most channel diverse is least likely to experience co-channel interference. WCETT of a path $p$ is defined as:

$$
W C E T T_{p}=(1-\beta) \cdot \sum_{l \in p} E T T_{l}+\beta \cdot \max _{1 \leq j \leq k} X_{j}
$$

where $\max X_{j}$ is the sum of ETTs on the most consumed channel and $k$ is the total number of channels available. $\beta$ is a tunable parameter used to alternate between the first term i.e. sum of ETTs which indicates path length and the second term i.e. $\max X_{j}$ which signifies channel diversity. The path with least WCETT value is chosen such that when $\beta$ is towards 0 , the WCETT value will reflect the path with least aggregate ETTs and when $\beta$ is towards 1, the WCETT will favor a path in which all channels have been equally used resulting in a channel diverse path.

WCETT performs better than ETX in multi-radio networks owing to its ability to exploit channel diverse paths and address co-channel interference. However WCETT has two important limitations. First limitation is WCETT's inability to adapt to changing network load. $\beta$ has been identified as critical to the performance of WCETT. However its value cannot be changed dynamically according to the network behavior and hence it has to persist with a certain predefined balance between path length and channel diversity, resultantly unable to take advantage of its flexibility. Second limitation is WCETT's channel diversity component fails to address inter-flow interference completely and only partially addresses intra-flow interference because it is unable to account for interference caused by the use of same channel in successive links along a path. Few metrics such as MIC [5], iAWARE [6] have been proposed to address the limitations of WCETT with regards to inter-flow interference. Both employ ETT in addition to other parameters and show improved performance over WCETT. However these metrics are more focused on interference-aware routing and do not address the aforementioned first shortcoming. This is especially the case for iAWARE as it employs a tunable parameter which remains constant and hence fails to find an effective way to alternate between metric components signifying path length and channel interference.

WCETT with Load Balancing (WCETT-LB) [7] modifies WCETT by introducing load balancing at mesh routers with the help of average queue length.

$$
W C E T T-L B_{p}=W C E T T_{p}+L_{p}
$$

where load $L_{p}$ of a path $p$ is given by:

$$
L_{p}=\sum_{\text {node } i \in p} \frac{Q L_{i}}{b_{i}}+\min (E T T) N_{i}
$$

$Q L_{i}$ is the average queue length at node $i, b_{i}$ is the transmission rate and $\min (E T T) N_{i}$ reflects traffic concentration at node $N_{i}$. WCETT-LB's performance has only been evaluated against hop count which fails to show whether it achieves any improvement over WCETT or not. Moreover it carries with it all the limitations of WCETT, including failure to switch between path length and channel diversity dynamically. Also WCETT-LB has only been analysed in a single-radio environment. 
Load-Aware Routing Metric (LARM) [8] also utilises ETT and queue information to form load balanced paths in a multiradio wireless mesh network.

$$
L A R M=(1-\beta) \cdot \sum_{j=1}^{k} C L(j)+\beta \cdot \max _{1 \leq j \leq k} C L(j)
$$

where Channel Load $C L$ of channel $j$ is defined as:

$$
C L(j)=\sum_{l \in \text { channelj }} E T T_{l} \cdot Q_{l}
$$

$Q_{l}$ is the average number of packets buffered on a link $l$ between two neighboring nodes and $k$ is the total number of channels available. First term in LARM shows accumulated load of occupied channels on the routing path whereas the second term reflects channel diversity. LARM struggles to show any significant performance improvement over WCETT while continuing to use a static $\beta$ value. It also fails to address the issue of inter-channel interference.

Recently proposed metrics such as Load-Aware Airtime Link Cost Metric [9] and Neighbourhood Load Routing (NLR) metric [10] use queue length in their metric considerations. Load-Aware Airtime has been evaluated against WCETT-LB and LARM but fails to show any substantial improvement while also using a static tunable parameter. NLR has been developed for single radio mesh networks and no evaluation is offered for multi-radio nodes.

\section{DYNAMIC WCETT (D-WCETT)}

\section{A. Problem of Static $\beta$}

The Dynamic WCETT (D-WCETT) consists of a number of modifications to WCETT metric and hence it is important to provide a brief overview of WCETT's original implementation before discussing our proposed extensions. WCETT was implemented on a source driven, proactive routing protocol called Multi-Radio Link Quality Source Routing (MR-LQSR). The protocol identifies all network nodes and computes the ETT values of all paths. Depending upon the value of $\beta$ it either favors shortest path or channel diverse path. The value of $\beta$ is static and cannot be changed once assigned to a network. Hence a wireless network using WCETT is either configured to favor shortest path $(\beta=0.1)$ or channel diverse paths $(\beta=0.9)$ or a compromise between the two $(\beta=0.5)$. This presents a drawback as WCETT cannot adapt to a changing network topology such as the one presented by a Hybrid WMN. Moreover it is also shown in [4] that when load levels are increased and the throughput is evaluated for different values of $\beta$, the maximum throughput is achieved using the lowest value of $\beta=0.1$. Therefore WCETT's performance is dependent upon the value of $\beta$ and results conclude that at high load levels, network throughput can be maximized by using lower values of $\beta$. Linking $\beta$ to a parameter that can accurately reflect the network load may result in better performance.

\section{B. Interface Queue length (IFQ)}

To calculate network load we have used interface queue length (IFQ) which is a parameter indicating the total number of packets in the queue of a channel belonging to a node [2]. The IFQ is a drop-tail buffer present at the MAC layer of IEEE 802.11 radios and contains outbound frames for the physical layer. A build up of frames indicates congestion due to high network traffic or low link quality. WMNs have a shared wireless medium which invites contention among nodes. This contention leads to congestion in the outbound IFQ of nodes. Queue lengths are properties of network nodes. Every network node possesses ingress and an egress queue in which incoming and outgoing packets are stored if the interface is unable to forward them immediately. The queue length gives an indication of the current state of the device: An empty queue is reflective of the fact that the device can process more traffic, whereas a full queue depicts a contented interface which cannot handle more packets. Usually, the queue length is small and as it becomes full, the node starts dropping packets. D-WCETT uses the IFQ as an indicator of link congestion. IFQ is indicative of a number of parameters including link quality, link load and external interference. The most significant advantage of using IFQ is that it is locally available at the node and hence can be used by nodes to make a decision about route selection without incurring additional communication overhead. Also IFQ can be calculated instantaneously making D-WCETT agile and able to adapt to dynamic networks. However it is usually desired that shortterm variations do not influence the value of a metric, as this could cause disproportionate adaptations of the metric and would increase the risk of self interference. Therefore metric measurements should be filtered over time by making use of a moving average. In D-WCETT the IFQ value is calculated as a moving average, filtered over a fixed interval of time called IFQ Window Size.

\section{Queue Discharge Interval (QDI)}

To cater for interfaces with dissimilar data rates, we divide the IFQ length by the bandwidth of the channel $(B W)$. This term is referred to as the Queue Discharge Interval $(Q D I)$.

$$
Q D I=\frac{I F Q}{B W}
$$

The $Q D I$ value represents the time required by a packet to remain in the IFQ before transmission. By normalizing the value it is ensured that the IFQ of different nodes with varying channel bandwidths are comparable.

\section{Integrating $\beta$ with Network Load}

Since $Q D I$ gives a real-time and accurate picture of the network load, we calculate $\beta$ such that when a link has a high value of $Q D I$, the $\beta$ values for calculating WCETT metric are lower. Hence D-WCETT does not have a constant predefined value of $\beta$ but a dynamic value, changing according to the level of congestion in the link. D-WCETT makes use of a simple relation to express the inverse relationship between $\beta$ and link load at a given instant.

$$
\beta=1-Q D I
$$

Whenever a route request is received at a node, the current value of link congestion on all available channels is obtained through the IFQ value. Links operating on different bandwidths are compensated to get QDI which is then normalized between 0 and 1 , where 0 indicates an empty queue and 1 indicates a full queue. Depending upon the value of QDI, a value 

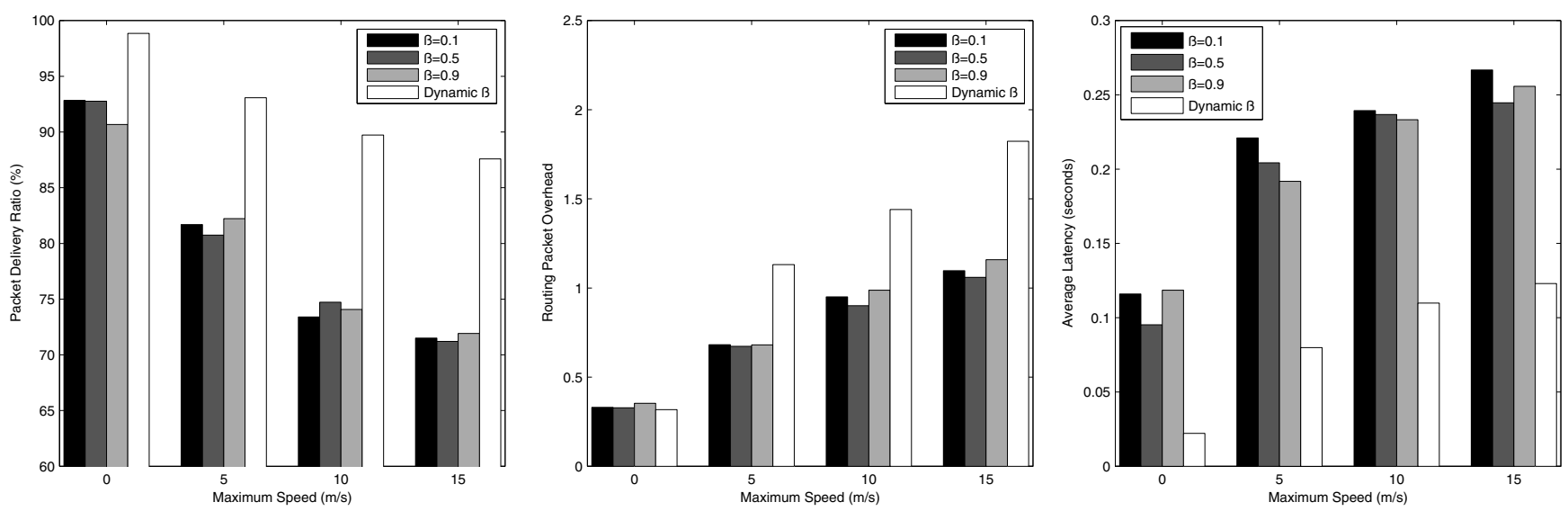

Fig. 1: Simulation results of varying the Mesh Clients' speed

of $\beta$ is selected according to (9). Using this $\beta$ value, DWCETT metric is calculated for all channels and route request is forwarded on the channel with the least D-WCETT value. In essence D-WCETT gives higher weight to path length (sum of ETTs) as compared to channel diversity when links are having higher load levels and vice versa.

\section{Simulation Results and Analysis}

\section{A. Simulation Environment}

The performance of D-WCETT has been evaluated through extensive simulations in NS-2 [11]. AODV [12] has been selected as the routing protocol owing to its suitability and applicability to Hybrid WMNs. Since WCETT metric was developed for link state routing protocol, a few adaptations have been made to AODV for WCETT to work on it [13] which are carried over to D-WCETT as well. The simulated network topology consists of 25 static Mesh Routers arranged in a $5 \times 5$ grid in a dense WMN covering an area of 1 square $\mathrm{km}$. Moreover 50 mobile Mesh Clients, each equipped with a single radio, are placed randomly in the simulation area. NS2 simulations were configured such that Mesh Routers were equipped with six $802.11 \mathrm{~b}$ radios tuned to orthogonal channels, hence additionally simulating a scenario where 802.11 a radios can be used which offer more non-overlapping channels than 802.11b. Concurrent UDP flows were established between randomly selected source and destination Mesh Client pairs. After experimenting with different values, we have found that IFQ Window Size of 0.1 seconds is best suited to our simulated network. The default simulation parameters are listed in Table 1. As we wanted to evaluate the behavior of Dynamic $\beta$, it was pertinent to test it against different static $\beta$ values. Hence three tests were conducted to evaluate the performance of D-WCETT routing metric in AODV protocol modified with multi-radio support and results were compared against WCETT (with static $\beta$ values of $0.1,0.5$ and 0.9 ) also implemented in multi-radio AODV.

\section{B. Performance Metrics}

The following three performance metrics were considered during the testing of D-WCETT:
TABLE I: Simulation Parameters

\begin{tabular}{|l|l|}
\hline & WCETT (with $\beta 0.1,0.5$ and 0.9 ) and \\
Evaluated Metrics & D-WCETT (Dynamic $\beta$ ) \\
Simulation Area & $1000 \times 1000 \mathrm{~m}$ \\
Simulation Time & 900 seconds \\
Propagation Model & Two-Ray Ground Reflection \\
Mobility Model for Mesh Clients & Random Waypoint \\
No. of Mesh Routers & 25 \\
No. of Mesh Clients & 50 \\
Traffic Type & CBR (UDP) \\
Packet Size & 512 bytes \\
Packet Transmission Rate & 32 pkts/sec \\
No. of Sources (Flows) & 30 flows \\
Max No. of Packets in IFQ & 50 packets \\
IFQ Window Size & $100 \mathrm{~ms}$ \\
& \\
&
\end{tabular}

- Packet Delivery Ratio: The ratio of total number of data packets successfully received and the total number of data packets transmitted.

- Routing Packet Overhead: The ratio of control packets generated and number of successfully received data packets.

- Latency: The mean time (in seconds) taken by data packets to reach their respective destinations.

In our evaluation, we have preferred to use Packet Delivery Ratio (PDR) over Goodput because the number of data bits transmitted do not remain same in Tests 2 and 3 and hence PDR gives a more accurate picture of routing efficiency in our simulated scenarios. Nevertheless we have also obtained the results of Goodput which are synonymous to the PDR results but could not be shown here due to limited space.

\section{Results and Analysis}

1) Test 1: Varying the Maximum Mesh Client Speed: In Test 1 , we have studied the effect of mobility on the routing performance and in doing that evaluated the metrics in a Static vs. Highly Mobile Network scenario as well. The maximum speed of the mesh clients was varied from $0 \mathrm{~m} / \mathrm{s}$ to $15 \mathrm{~m} / \mathrm{s}$ and thirty 128kbps CBR flows were maintained between random mesh client pairs. The results for WCETT (with $\beta$ values of 

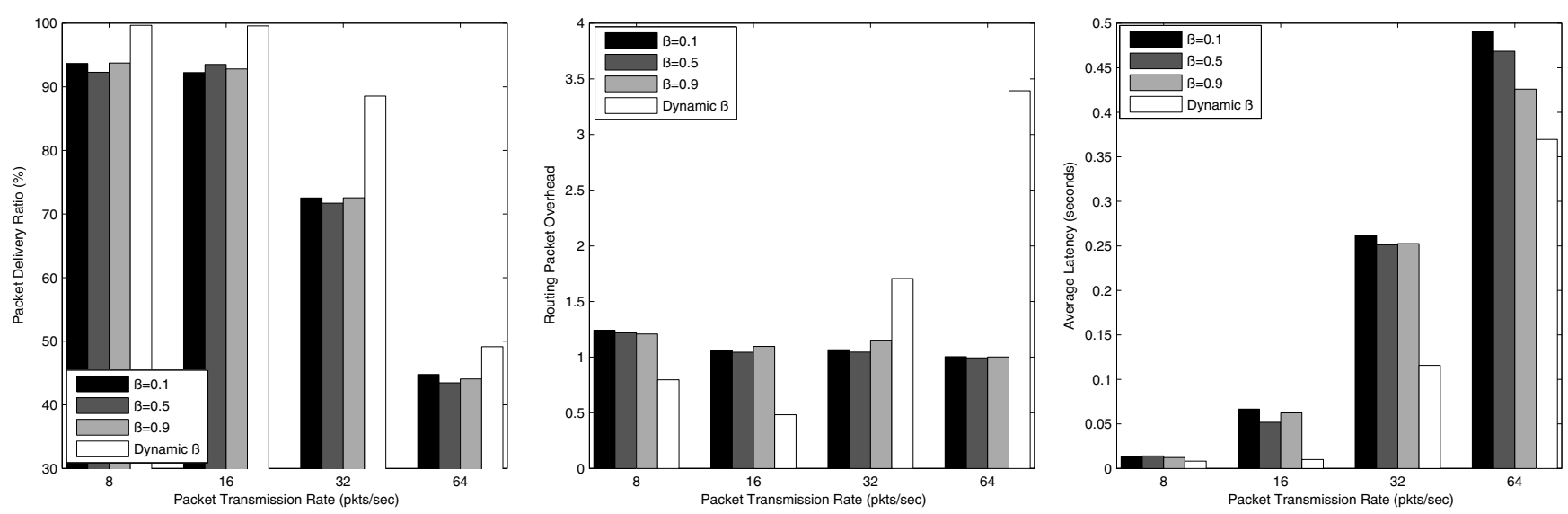

Fig. 2: Simulation results of varying the packet transmission rate

0.1, 0.5 and 0.9) and D-WCETT (with Dynamic $\beta$ ) are shown in Fig. 1. D-WCETT achieves significantly higher PDR at all speeds as compared to all $\beta$ values of WCETT with the improvement becoming more evident as the network becomes highly mobile. This improvement of D-WCETT is owing to its ability to change the value of $\beta$ according to network load. If the network is facing congestion at a particular node, DWCETT sets a lower value of $\beta$, which favors shorter paths, resulting in higher PDR. But at the same time areas within the network which are least congested and for whom channel diverse paths would yield optimum results are assigned higher values of $\beta$ by the D-WCETT metric.

The routing packet overhead remains the same for WCETT and D-WCETT at $0 \mathrm{~m} / \mathrm{s}$ speed. But as the speed of mesh clients increases, the overhead begins to show in case of DWCETT. The reason behind the increased overhead in DWCETT's case is the forwarding of subsequent route request (RREQ) packets having lower (and better) WCETT values. At a particular instant a node may discard a RREQ on a particular channel if another channel is found to be less congested. But the same channel may be more favorable after some time and hence a subsequent RREQ would be allowed to propagate through that channel. Hence the number of control packets generated for each data packet would tend to increase. The most notable improvement of D-WCETT over the three values of $\beta$ in WCETT is in terms of the average network latency. D-WCETT considerably outperforms all values of $\beta$ at all mesh client speeds. In D-WCETT paths are created having the least number of packets in interface queues. Thus after the creation of a path from source to destination, data packets traverse through the network in less time as they are navigated through least filled queues.

2) Test 2: Varying the Packet Transmission Rate: In Test 2 the packet transmission rate was varied from 8 pkts/sec to $64 \mathrm{pkts} / \mathrm{sec}$, and thirty CBR flows were maintained between random mesh client pairs moving at a speed of $15 \mathrm{~m} / \mathrm{s}$. The corresponding performance metrics for WCETT (with $\beta$ values of 0.1, 0.5 and 0.9) and D-WCETT (with Dynamic $\beta$ ) are shown in Fig. 2. The results indicate that D-WCETT with its Dynamic $\beta$ achieves better PDR as compared to all static $\beta$ values at packet transmission rates of 8,16 and $32 \mathrm{pkts} / \mathrm{sec}$ whereas at $64 \mathrm{pkts} / \mathrm{sec}$ it becomes comparable with the rest. This is because at such high transmission rates $(256 \mathrm{kbps}$ per flow), all links become equally congested eliminating the possibility of any improvement.

The routing overhead of D-WCETT remains slightly lower than WCETT at 8 and $16 \mathrm{pkts} / \mathrm{sec}$ but at $64 \mathrm{pkts} / \mathrm{sec}$, it registers a significant increase. This is because of the forwarding of subsequently better RREQ which when combined with such high packet sending rates, leads to a storm of control packets. In terms of latency D-WCETT performs better than all values of $\beta$ at all transmission rates. The delay experienced by packets in D-WCETT metric is significantly low till $32 \mathrm{pkts} / \mathrm{sec}$ but becomes comparable at higher transmission rates. In DWCETT paths are created having the least number of packets in interface queues and hence packets take less time to reach their destinations.

3) Test 3: Varying the Number of Flows: In Test 3 the simultaneous number of connections between nodes was varied from 10 to 50 connections. Speed of mesh clients was set to 1 $\mathrm{m} / \mathrm{s}$ and packet transmission rate was $32 \mathrm{pkts} / \mathrm{sec}$ for each flow. The corresponding performance metrics for WCETT (with $\beta$ values of $0.1,0.5$ and 0.9 ) and D-WCETT (with Dynamic $\beta$ ) are shown in Fig. 3. For a lightly loaded network with only 10 concurrent flows, all metrics perform equally well. However, as soon as the load is increased beyond 20 flows, the improvement of D-WCETT becomes apparent. With an aggregate network load of more than $6 \mathrm{Mbps}(50 \times 128 \mathrm{kbps})$ D-WCETT is still able to maintain a reasonable PDR of almost $85 \%$. Another important point to note is that with increase in number of flows, permanently favoring a lower static value of $\beta$ does not necessarily yield better performance as the results indicate that all static $\beta$ values $(0.1,0.5$ and 0.9$)$ achieve comparable PDR values. This strengthens our argument that keeping $\beta$ dynamic yields better results as the network alternates between path length and channel diversity according to changing network conditions.

The routing overhead of D-WCETT remains lower than others at 10 and 20 flows. It becomes slightly higher at 30 and 40 number of flows and increases significantly at 50 flows. Even at 30 number of flows, the network poses 

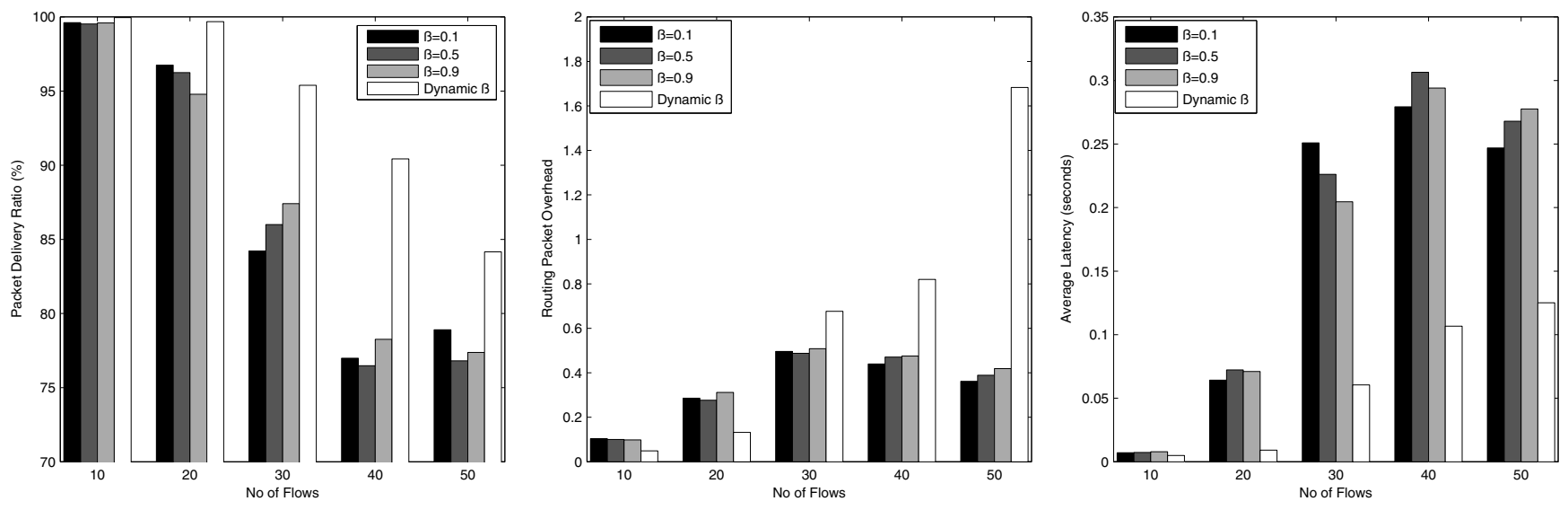

Fig. 3: Simulation results of varying the number of concurrent flows

a significant data rate of $3.75 \mathrm{Mbps}$, resulting in a highly congested network. Nodes drop packets and routes start to become invalid, necessitating new route discoveries leading to generation of more control packets. Dynamic $\beta$ aggravates the situation further as frequent changes in IFQs lead to a continuously changing metric value, which leads to frequent route changes. Hence D-WCETT results in higher routing overhead with increasing number of flows. Still the paths that are eventually formed by D-WCETT have least congested queues which is reflected in decreased latency values for Dynamic $\beta$. Data packets in D-WCETT need half as less time to reach their destination nodes as compared to WCETT at all static $\beta$ values.

\section{CONCLUSION}

Hybrid WMNs present a promising technology for robust and reliable communication. This paper proposes D-WCETT, a routing metric with load aware, dynamic path selection in multi-radio Hybrid WMNs. The proposed metric is an extension of WCETT metric and takes transmission time and channel diversity into account while at the same time assigning weight to them on the basis of link congestion. The paper removes the limitation of WCETT, which was unable to cater for the changing network topology, while dynamically building routes based on the prevalent network load information. The network load is measured through interface queue lengths of each channel at the node. The IFQ value is then normalized to take into account links of different bandwidths and $\beta$ is calculated from this parameter which is then used to compute the value of D-WCETT metric. This calculation of $\beta$ is dynamic and reflective of the current channel load. Based upon the value of this metric, a node decides on which interface to forward the route request. Owing to its dynamic calculation, D-WCETT is able to select least congested links with less number of packets in queues. The results clearly indicate the superior performance of the D-WCETT metric against different static values of $\beta$ from WCETT metric when implemented on AODV protocol in a multi-radio Hybrid WMN. Higher packet delivery ratio and better latency indicate significant improvement of D-WCETT over all static $\beta$ values of WCETT. However due to its characteristic of adapting to current network conditions, D-WCETT incurs additional routing overhead. The better performance of D-WCETT is attributed to the integration of $\beta$ with network load. In future, we plan to implement D-WCETT metric on a hardware test bed. Moreover a comparative analysis of the proposed load dependent routing metric D-WCETT with other contemporary load balancing metrics can be performed.

\section{REFERENCES}

[1] I. F. Akyildiz, X. Wang, and W. Wang, "Wireless mesh networks: a survey," Computer Networks, vol. 47, no. 4, pp. 445-487, 2005.

[2] R. Baumann, S. Heimlicher, M. Strasser, and A. Weibel, "A survey on routing metrics," TIK Report, vol. 262, 2007.

[3] D. S. De Couto, D. Aguayo, J. Bicket, and R. Morris, "A highthroughput path metric for multi-hop wireless routing," Wireless Networks, vol. 11, no. 4, pp. 419-434, 2005.

[4] R. Draves, J. Padhye, and B. Zill, "Routing in multi-radio, multi-hop wireless mesh networks," in Proc. of the 10th Annual Int. Conf. on Mobile Computing and Networking, 2004, pp. 114-128.

[5] Y. Yang, J. Wang, and R. Kravets, "Designing routing metrics for mesh networks," in Proc. of IEEE Workshop on Wireless Mesh Networks (WiMesh), 2005.

[6] A. P. Subramanian, M. M. Buddhikot, and S. Miller, "Interference aware routing in multi-radio wireless mesh networks," in Proc. of 2nd IEEE Workshop on Wireless Mesh Networks (WiMesh), 2006, pp. 55-63.

[7] L. Ma and M. K. Denko, "A routing metric for load-balancing in wireless mesh networks," in Proc. of 21st Int. Conf. on Advanced Information Networking and Applications Workshops, vol. 2, 2007, pp. 409-414.

[8] A.-N. Le, D.-W. Kum, Y.-Z. Cho, and I.-S. Lee, "LARM: A load-aware routing metric for multi-radio wireless mesh networks," in Proc. of IEEE Int. Conf. on Advanced Technologies for Communications, 2008, pp. 166-169.

[9] J. Y. Choi and Y.-B. Ko, "Multi-path routing with load-aware metric for tactical ad hoc networks," in Proc. of IEEE Int. Conf. on Information and Communication Technology Convergence, 2010, pp. 370-375.

[10] L. Zhao, A. Y. Al-Dubai, and G. Min, "An efficient neighbourhood load routing metric for wireless mesh networks," Simulation Modelling Practice and Theory, vol. 19, no. 6, pp. 1415-1426, 2011.

[11] NS, "The Network Simulator," http://www.isi.edu/nsnam/ns/, 1989.

[12] C. E. Perkins and E. M. Royer, "Ad-hoc on-demand distance vector routing," in Proc. of 2nd IEEE Workshop on Mobile Computing Systems and Applications, 1999, pp. 90-100.

[13] A. A. Pirzada, M. Portmann, R. Wishart, and J. Indulska, "Safemesh: A wireless mesh network routing protocol for incident area communications," Pervasive and Mobile Computing, vol. 5, no. 2, pp. 201-221, 2009 . 